\title{
Measurements and Modeling of Transient Blood Flow Perturbations Induced by Brief Somatosensory Stimulation
}

\author{
Reswanul Khan ${ }^{1,2}$, Andrew K. Dunn ${ }^{3}$, Timothy Q Duong ${ }^{4}$ and David Ress ${ }^{*, 1}$ \\ ${ }^{I}$ Psychology, Neurobiology, Center for Perceptual Systems, Imaging Research Center, University of Texas at Austin, \\ USA; ${ }^{2}$ Department of Physics, University of Texas at Austin, USA; ${ }^{3}$ Department of Biomedical Engineering, University \\ of Texas at Austin, USA; ${ }^{4}$ Research Imaging Institute, Departments of Ophthalmology, Radiology and Physiology, \\ University of Texas Health Science Center, San Antonio, TX, USA
}

\begin{abstract}
Proper interpretation of BOLD fMRI and other common functional imaging methods requires an understanding of neurovascular coupling. We used laser speckle-contrast optical imaging to measure blood-flow responses in rat somatosensory cortex elicited by brief ( $2 \mathrm{~s}$ ) forepaw stimulation. Results show a large increase in local blood flow speed followed by an undershoot and possible late-time oscillations. The blood flow measurements were modeled using the impulse response of a simple linear network, a four-element windkessel. This model yielded excellent fits to the detailed time courses of activated regions. The four-element windkessel model thus provides a simple explanation and interpretation of the transient blood-flow response, both its initial peak and its late-time behavior.
\end{abstract}

Keywords: brain, hemodynamics, vasodilation, oxygen transport, microvessels, brain mapping.

\section{INTRODUCTION}

A detailed understanding of the neurovascular response in the brain is of critical value to science and medicine. Fundamentally, the neurovascular response is tightly linked in basic operation of the mind and brain. It seems likely that we will need a deeper understanding of vascular processes to grasp the strategies of neural computation. The success, in fact, of hemodynamic imaging techniques such as fMRI highlights the need for better understanding of neurovascular coupling. Specifically, knowledge of the vascular impulse response evoked by a brief (few seconds) stimulation would be useful. This is known as the hemodynamic response function (HRF). The HRF is formed by changes in oxygen uptake and blood flow. Measurements in cats $[1,2]$ and in rats, e.g., [3], indicate that the HRF typically exhibits a 3phase response: initial delay or dip lasting $2-3$ s, hyperoxic peak at 5-8 $\mathrm{s}$, and undershoot with frequent ringing. Because the brain is continually active, it is likely that such transient hemodynamic events occur continually throughout the brain. In order to understand the healthy operation of the brain, its pathophysiology, and the mechanisms of hemodynamic imaging, there is a need to better understand the hemodynamic processes that give rise to the HRF.

A number of models have been proposed for the dynamics of the HRF. The "balloon model" is the most popular $[4,5]$. It explains the HRF based on flow and volume changes in the intra- and extravascular compartments with non-linear compliance. The volume changes were postulated to occur in venules and veins, as the global

\footnotetext{
*Address correspondence to this author at the UT Austin Imaging Research Center, 3925B West Braker Lane, R9975, Austin, TX 78759 USA; E-mail: ress@ mail.utexas.edu
}

compliance of venous system is known to be substantial [68]. A similar form for this model utilizes a lumped-element network analogy that includes a non-linear compliant element $[9,10]$. Such models have become increasingly sophisticated and complex [11-13]. However, recent experiments have not shown much venular dilation; volume increases seem mostly to occur on the arterial side of the system $[14,15]$. Accordingly, there is a need to revisit theoretical models for the HRF and its associated flow perturbation.

We propose a linear flow model, the four-element windkessel (FEW). This model, when combined with a convection-diffusion description of oxygen transport [16], provided excellent fits to tissue-oxygen measurements $[1,2]$. In those results, the flow damping time constant suggested strongly underdamped responses. It is well known that the flow response of the HRF is mediated by the action of smooth-muscle fibers on the larger arterioles, and there is substantial evidence that pericytes modulate flow in small arterioles [17-20]. The flow response itself has been characterized extensively using such means as in vivo laserDoppler flowmetry, e.g., [21-23], and laser speckle contrast imaging, e.g., [24-26]. Here we applied the FEW model to describe speckle-contrast measurements corresponding to flow-speed perturbations induced in rat somatosensory cortex by brief forepaw stimulation.

\section{MATERIALS AND METHODOLOGY}

\section{Model}

We model the flow perturbation using the FEW, a lumped linear equivalent-circuit model for the blood flow dynamics (Fig. 1A) [16]. The inertive element, $I$, models Newton's $2^{\text {nd }}$ law regarding the flow of blood in the pial arteries. The series resistance, $R_{1}=R_{1 \mathrm{a}}+R_{1 \mathrm{~b}}$, represents 
frictional losses associated with the parallel combination of all downstream flow through the fractal vascular tree. The elements $C$ and $R_{2}$ represent the lossy compliance of the downstream venous vasculature. In steady state, and neglecting cardiac pulsatility, there is a constant pressure at point $\mathbf{T}$, which represents the input to our model. The upstream vasculature, where flow perturbations are actively driven, couples resistively to the larger network, but the load resistance $R_{A}$ is much greater than $R_{1}$ and $R_{2}$, so it does not perturb the global dynamics. The transient behavior of this system is modeled by the switch across $R_{1 b}$, which briefly closes to simulate a vasodilation that decreases the upstream vascular resistance. Because there was steady-state flow through $I$, the vasodilation creates a brief positive pressure fluctuation at $\mathbf{T}$, which is described by the impulse response of the network.

Because this system is linear, its impulse response fully characterizes its behavior. The flow perturbation impulse response has two forms depending upon the time constants of the system (Eqn.1).

$$
U_{1}(t)=\left\{\begin{array}{l}
U_{10} e^{-t / \tau} \sin (2 \pi f t) \\
U_{10} e^{-t / \tau} \sinh (2 \pi f t)
\end{array}\right.
$$

The first form is under-damped and the second over-damped (Fig. 1B). The flow perturbation is specified by three parameters: frequency $f$, damping time $\tau$, and amplitude $U_{10}$.

\section{Animal Procedure}

Three male Sprague-Dawley rats (250-350 g) rats were anesthetized with halothane $(2 \%)$ and each animal was placed in a stereotaxic frame (Kopf Instruments, Tujunga, CA). A heating pad (WPI ATC1000) was used to maintain body temperatures of $37{ }^{\circ} \mathrm{C}$. Then, in each rat, on the left hemisphere around the somatosensory cortex, a $4 \mathrm{~mm} \times 4$ $\mathrm{mm}$ portion of skull was thinned to transparency using a dental burr and then removed. A well was formed around the craniotomy using dental cement, which was then filled with silicone gel to improve visibility. Following surgery, animals were artificially ventilated with $1.5 \%$ halothane, $70 \% \quad \mathrm{~N}_{2} \mathrm{O}$ and $30 \% \mathrm{O}_{2}$, using procedures described previously [27].

All animal protocols were approved by the Animal Care and Use Committees of the University of Texas at Austin.

\section{Instrumentation}

A laser diode $(808 \mathrm{~nm}, 30 \mathrm{~mW})$ and CMOS camera (Basler 602f, $610 \times 490$ pixels) were used to acquire laser speckle contrast imaging (LSCI) of blood flow in the cerebral cortex of each rat (Fig. 2) [25]. The laser diode was placed approximately $20 \mathrm{~cm}$ away from the animal at a $45^{\circ}$ angle such that the area of interest was illuminated evenly. The scattered light was optically magnified between $1 \times$ and $3 \times$ depending on the animal. It was then captured by the camera at a rate of 100 frames/second and an exposure time of $5 \mathrm{~ms}$. The acquisition was digitally recorded using custom software.

\section{Experiment}

The rat's forepaw was electrically stimulated for $2 \mathrm{~s}$ starting $0.5 \mathrm{~s}$ after the trial onset. To induce somatosensory activity via electrical stimulation, needles were inserted between the digits, which were connected to a stimulus isolation unit (World Precision Instruments, Sarasota, FL). Current pulses of 1-2 mA were applied for $300 \mu$ s at a rate of $3 \mathrm{~Hz}$ for 2 seconds. The current level was adjusted to evoke a slight, visible twitch of one-or-more forepaw digits [27].

Forty trials with an inter-stimulus interval of $15 \mathrm{~s}$ were performed while image data were recorded at a rate of 100 frames/sec, then processed and displayed in real time [28].The processed data were recorded for later analysis.

\section{Speckle Contrast Image Analysis}

The speckle contrast image is defined as the ratio of the standard deviation to the mean intensity in a small region of the image, $K \equiv \sigma_{\mathrm{s}} /\langle\mathrm{I}\rangle$. The region must be chosen large enough that there is sufficient contrast to noise ratio, but not so large as to adversely limit our spatial resolution. With this in mind, a $7 \times 7$ region of pixels was empirically determined to balance these requirements; each pixel is $\sim 9 \mu \mathrm{m}$. A speckle contrast map was computed from the raw image using custom written softwareand downsampled to 10 frames/sec to improve signal-to-noise ratio (SNR) [25, 27].

\section{Flow Computation}

The speckle-contrast images were then converted to images of relative correlation times $\tau_{\mathrm{c}}$, $K=\sqrt{\tau_{c}\left(1-e^{-2 T / \tau_{c}}\right) / 2 T}$ where $K$ is the speckle contrast and $T$ is the camera exposure time [29]. We used the temporalmean of the correlation-time images as a structural image underlay for our results because of their apparent congruence with the vasculature (Figs. 2A, B). Larger blood vessels have higher blood speeds, yielding smaller correlation times, so the vasculature shows up as darker regions in the images.
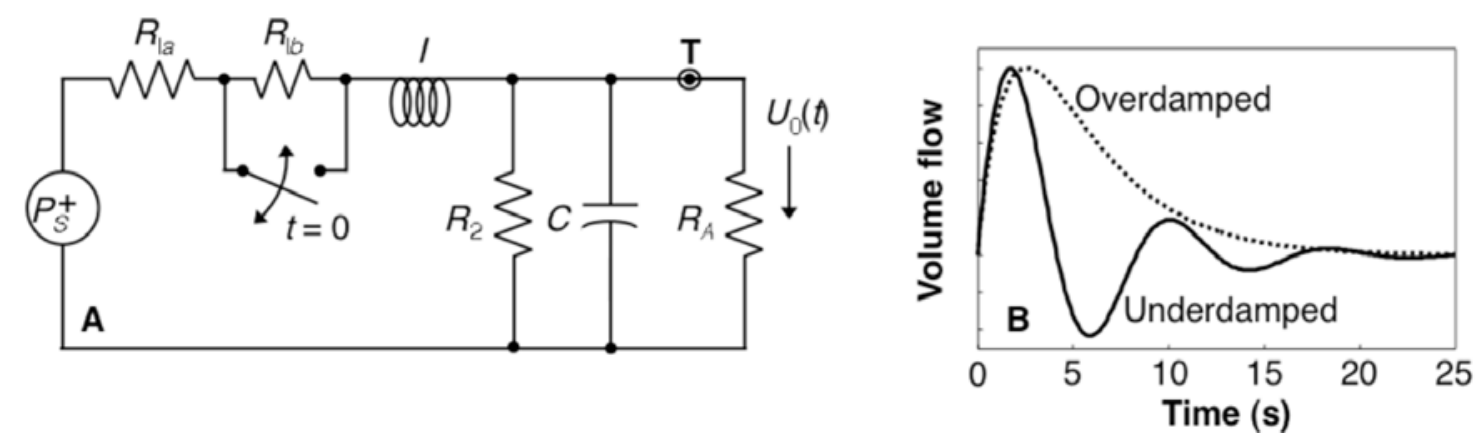

Fig. (1). (A) FEW network; (B) impulse response 

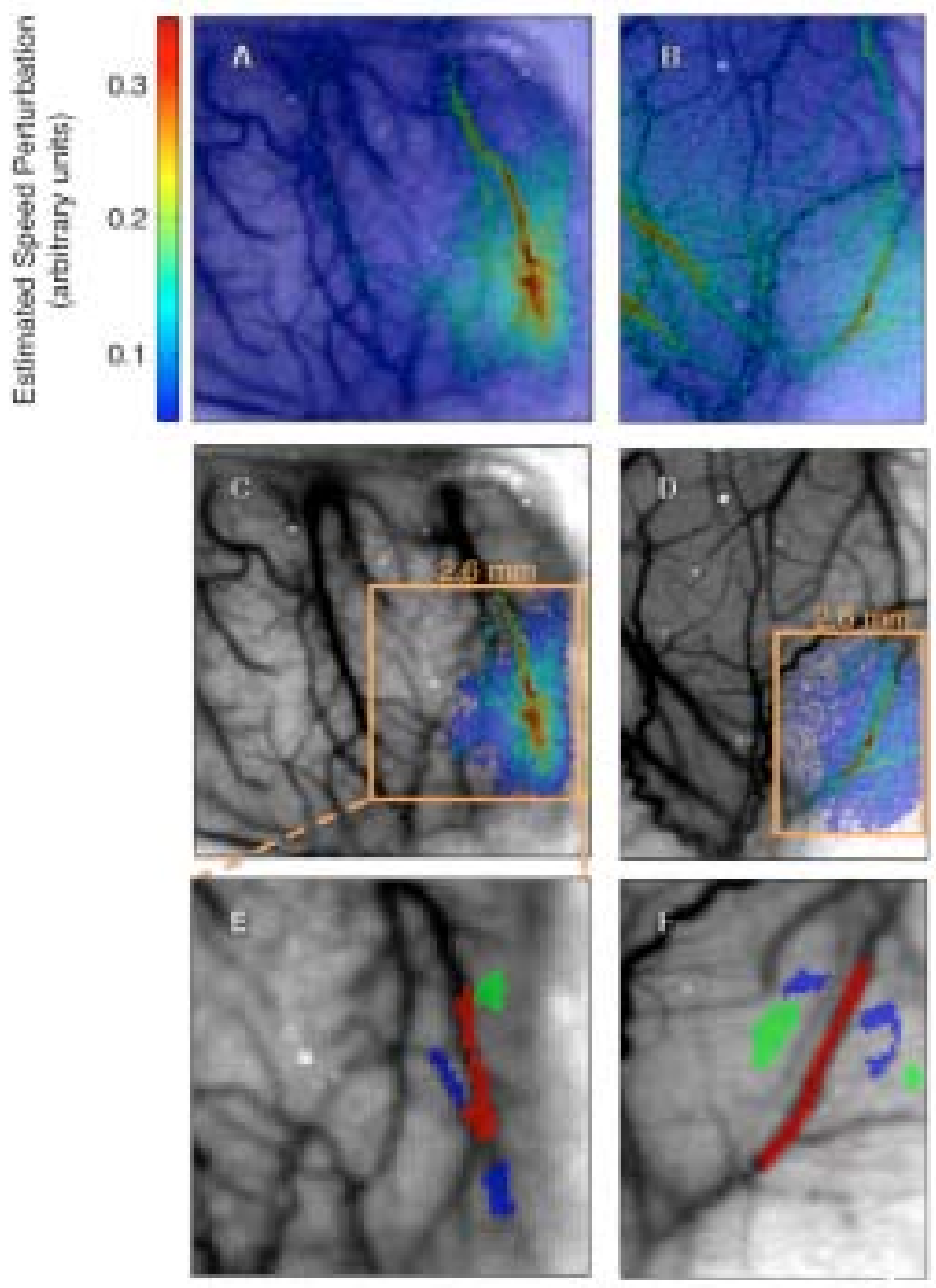

Fig. (2). Measured flow perturbations in two animals: across the entire image (A, B), and restricted to a region where CNR $\geq 8$ in animal 1 (C), and CNR $\geq 3.2$ in animal 2 (D). Selected ROIs in this region (E,F): parenchyma, small blood vessels (blue), large blood vessels (red).

To facilitate comparison with our flow model, we converted these values to a quantity related to blood speed. Since blood speeds may be assumed to be roughly inversely proportional to correlation times, we defined the normalized blood speed $v \equiv k / \tau_{\mathrm{c}}$, where $k$ is a proportionality constant [30]. We must be careful not to explicitly associate this with the local physical blood speed, as this assumption neglects numerous contributions to speckle contrast such as multiple scattering and penetration-depth effects [31]. To emphasize this loose connection, we denote the units of blood speed to be arbitrary. However, we chose $k$ such that the average value of $v$ in regions of parenchyma equaled 0.7 , the average blood speed in rat cerebral cortex parenchyma in $\mathrm{mm} / \mathrm{s}$. This provided a rough sense of the data in terms of real units [32]. Using this relationship, we created a spatial and temporal map of relative blood flow speed $(454 \times 433$ pixels $\times 15 \mathrm{~s} \times 40$ trials). This was then spatially down sampled over a $4 \times 4$ region to bring the dimensions to $113 \times 108$ pixels with size $36 \mu \mathrm{m}$.

To estimate the blood-flow response, we convolved the impulse response $U_{1}(t)$ (Fig. 1B) with a stimulus function, a 2 -s-duration rectangular function ( $\Pi$ ) centered at $t=1.5 \mathrm{~s}$ :
$F(t)=\Pi[(t-1.5 \mathrm{~s}) / 2] *\left[\theta(t-0.5 \mathrm{~s}) U_{1}(\mathrm{t})\right] / 20$, where $\theta$ is a unit step function. This convolution served to approximate the vasodilation effects of the 2-s-duration somatosensory stimulus. The convolution stretched out the waveform causing an apparent $\sim 0.5 \mathrm{~s}$ delay to the initial perturbation response. Although this flow perturbation is often strongly damped, with a 15-s ISI the perturbation may not decay to zero at the end of each trial. We included these effects by applying our model to a trial-averaged data set $v_{1}(x, y, t)=\frac{1}{N} \sum_{n=0}^{N-1} F(t-n L)$, where $x$ and $y$ refer to the coordinates of the pixel, $t$ is time after trial onset, $N$ is the total number of trials, and $L$ is the trial length. Total blood speed is found by adding the baseline speed $v_{0}$ to the perturbation, $v(x, y)=v_{0}(x, y)+v_{1}(x, y, t)$.

We restricted our data analysis to pixels where activity exceeded a minimum contrast-to-noise ratio (CNR). The noise was estimated by computing the standard error about the mean for $v$ across trials and then averaged over the last third (5 s) of each trial. Contrast, which is the perturbation 
amplitude in this case, was estimated by taking the maximum blood speed in each pixel and subtracting the baseline blood speed (Fig. 2A). The baseline blood speed $v_{0}$ was computed by taking the mean of the normalized blood speeds in the last half-second of each trial. We then restricted our analysis to a contiguous region of pixels that exceeded a particular threshold (Figs. 2C, D). Within this region, we defined three compartmental regions-of-interest (ROIs) to illustrate response time series in large vessels, small vessels, and parenchyma (Figs. 2E, F).

To obtain fits we used MATLAB's ls qcurvefit routine to perform non-linear optimization [33], varying the parameters $U_{10}, f$, and $\tau$ to minimize the RMS error between the measurement and the model. We determined fit quality using the fraction of the variance explained, FOVE $\equiv 1-\sigma_{\text {fit - data }}^{2} /$ $\sigma_{\text {data }}{ }^{2}$. We considered a fit to be successful if FOVE> $1-$ $1 / \mathrm{CNR}_{\min }$, where $\mathrm{CNR}_{\min }$ was the smallest $\mathrm{CNR}$ in the ROI (Fig. 2C-D)

The experimental error per trial was defined to be the time averaged standard error of the mean over trials and is plotted as dotted lines in Fig. (3).

\section{RESULTS}

Good fits (FOVE > 80\%) were obtained for all of the ROI time series data (Table 1 and Fig. 3). Mild ringing was observed in our fits to all of the sample ROIs in animals 1 and 3. All ROI fits in this animal were underdamped with significant undershoot. The fits observed in animal 3 were largely qualitatively similar to those observed in animal 1, and will not be further presented except to note a few relevant differences.

Blood flow in animal 2 was distinctly different from the other two animals. FOVE was substantially smaller. The reduced fit quality was apparently caused by the presence of a $\sim 0.2 \mathrm{~Hz}$ underdamped wave superimposed on a more typical strongly damped waveform. In addition, the hyperoxic peak exhibited a shorter duration. Fits for this animal showed less ringing, particularly for the large vessel and parenchymal compartments, but FOVE was smaller because the fits did not model the additional underdamped component.
We also fit our model to all of the pixel time series that satisfied a minimum CNR threshold within the activated regions illustrated in Fig. 1. As noted previously, data from animal 2 had a much lower fit quality than the other two animals because of the apparently superimposed, stimulusevoked ringing. The CNR threshold was therefore chosen to be less than in the other animals to produce an ROI that was similar in size to that of the other two animals. Mean parameter values for $A, f$ and $\tau$ for thosepixel time series that were successfully fitare summarized in Table 2 . Fit frequency was similar in all animals. Damping time varied substantially, with the largest values (greatest ringing) in animal 3, while animal 2 exhibited the smallest values (greatest damping).

There was a strong positive correlation between perturbation amplitude and baseline blood speed $(R=0.78$, $p<10^{-16}$ in Fig. 4A and $R=0.77, p<10^{-16}$ in Fig. 4C). Spatially, the perturbation amplitude appeared to largely follow the vasculature (Figs. 4B, D). The largest amplitudes were in one-or-two large blood vessels and then appeared to diminish toward their vascular periphery.

In all animals, the frequency parameters exhibited a bimodal distribution (Figs. 5A, C). In animal 1, about $95 \%$ of the responsive pixels were better fit to an underdamped response with a mean frequency of $0.093 \pm 0.016 \mathrm{~Hz}$. About $5 \%$ were better fit to nearly critically damped responses; mean frequency in the lower mode is $0.0082 \pm 0.0051 \mathrm{~Hz}$. The mean frequencies in animal 2 were significantly higher in both the upper and the lower modes, $0.17 \pm 0.02 \mathrm{~Hz}$ and $0.014 \pm 0.008 \mathrm{~Hz}$ respectively. The strongly damped responses all corresponded to low baseline blood speed, suggesting that they occurred largely in parenchyma $\left(v_{0}<1\right)$. There was a negative trend of frequency with flow speed for the high-frequency mode in animal $1(R=-0.14, p<0.02)$. Animal 2 showed a small positive trend in the upper mode $(R=0.24, p<0.02)$. The frequency parameter showed a spatial distribution without a clear compartmental partition (Figs. 5B, D). In animal 1, the more strongly damped responses appeared mostly toward the right edge of the image, close to the edge of the craniotomy, while in animal 2 they were more central. The two modes seemed to occur largely in spatially distinct regions of the image.

Table 1. Fit Parameters for Vascular Compartment ROIs in the Three Animals

\begin{tabular}{|c|c|c|c|c|}
\hline \multirow[t]{2}{*}{1} & Parenchyma & 97.4 & 0.10 & 2.37 \\
\hline & Large Vessel & 99.2 & 0.094 & 1.74 \\
\hline 2 & Large Vessel & 83.0 & 0.0061 & 0.82 \\
\hline \multirow[t]{2}{*}{3} & Parenchyma & 98.0 & 0.076 & 2.57 \\
\hline & Small Vessel & 97.9 & 0.080 & 2.51 \\
\hline
\end{tabular}



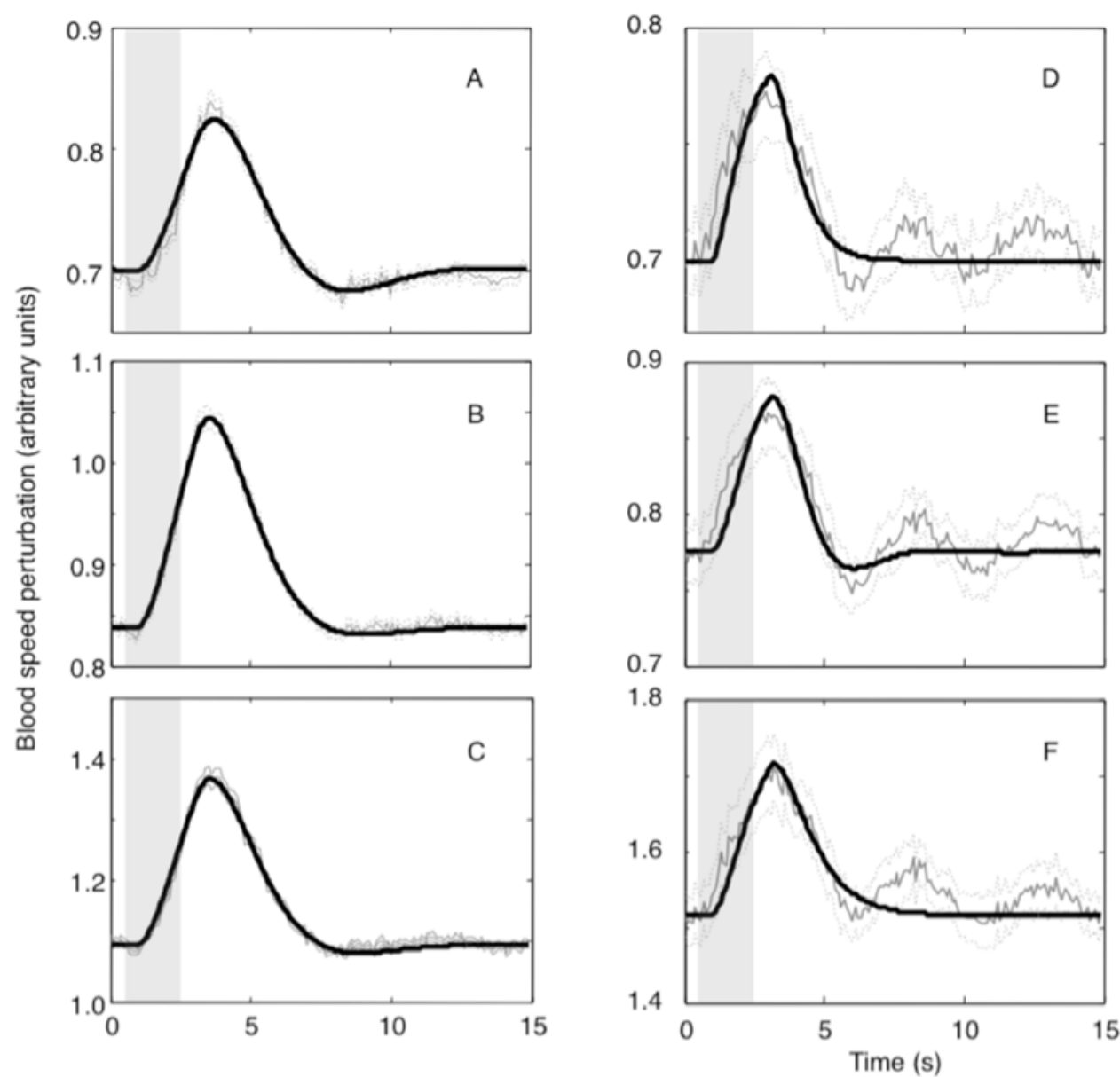

Fig. (3). Best fits to the mean time series in the three vascular regions shown for animal 1 in Fig. 1E: (A) parenchyma; (B) small vessels; (C) large vessels. Likewise (D), (E) and (F) for animal 2 in Fig. 1F. Gray column indicates stimulus period.

Table 2. Fit parameters, median \pm standard deviation, for the activated regions illustrated in Fig. (1)

\begin{tabular}{|c|c|c|c|c|c|c|}
\hline Animal & $\mathbf{C N R}_{\min }$ & Fit quality (\%) & Amp. mod. (\%) & $\boldsymbol{A}$ (arb. units) & $\boldsymbol{f}(\mathbf{H z})$ & $\boldsymbol{\tau}(\mathbf{s})$ \\
\hline 1 & 8 & 88.7 & $21.0 \pm 4.9$ & $0.169 \pm 0.054$ & $0.090 \pm 0.024$ & $1.89 \pm 0.42$ \\
\hline 2 & 3.2 & 29.0 & $14.0 \pm 2.7$ & $0.115 \pm 0.036$ & $0.107 \pm 0.077$ & $1.39 \pm 0.90$ \\
\hline 3 & 12 & 99.3 & $26.9 \pm 4.0$ & $0.222 \pm 0.052$ & $0.077 \pm 0.006$ & $2.53 \pm 0.24$ \\
\hline
\end{tabular}

\begin{tabular}{|c|c|c|c|c|c|c|}
\hline Animal & $\mathbf{C N R}_{\min }$ & Fit quality (\%) & Amp. mod. (\%) & $\boldsymbol{A}$ (arb. units) & $\boldsymbol{f ( H z )}$ & $\boldsymbol{\tau}(\mathbf{s})$ \\
\hline \hline 1 & 8 & 88.7 & $21.0 \pm 4.9$ & $0.169 \pm 0.054$ & $0.090 \pm 0.024$ & $1.89 \pm 0.42$ \\
\hline 2 & 3.2 & 29.0 & $14.0 \pm 2.7$ & $0.115 \pm 0.036$ & $0.107 \pm 0.077$ & $1.39 \pm 0.90$ \\
\hline 3 & 12 & 99.3 & $26.9 \pm 4.0$ & $0.222 \pm 0.052$ & $0.077 \pm 0.006$ & $2.53 \pm 0.24$ \\
\hline
\end{tabular}

A weak but highly significant positive correlation was found between damping time and blood speed in both animals $\left(R=0.20, p<6 \times 10^{-11}\right.$, Fig. 6B; $R=0.22, p<1 \times 10^{-13}$, Figs. 6A, C). Therefore, higher caliber vessels exhibited greater ringing. However, at lower blood speeds (parenchyma) we observed the widest range of damping time constants and some regions exhibited substantial undershoot (Fig. 3A). The damping time constant also showed a clear spatial trend, decreasing from upper left toward lower right in animal 1 (Fig. 6B) and from the upper right toward the lower left in animal 2 (Fig. 6D).

\section{DISCUSSION}

Flow changes (blood speed) had a mean value of $20.6 \%$, which is similar to other measurements, e.g., the $\sim 30 \%$ flow changes obtained by positron emission tomography and MRI $[34,35]$. The linear relationship between the perturbation 

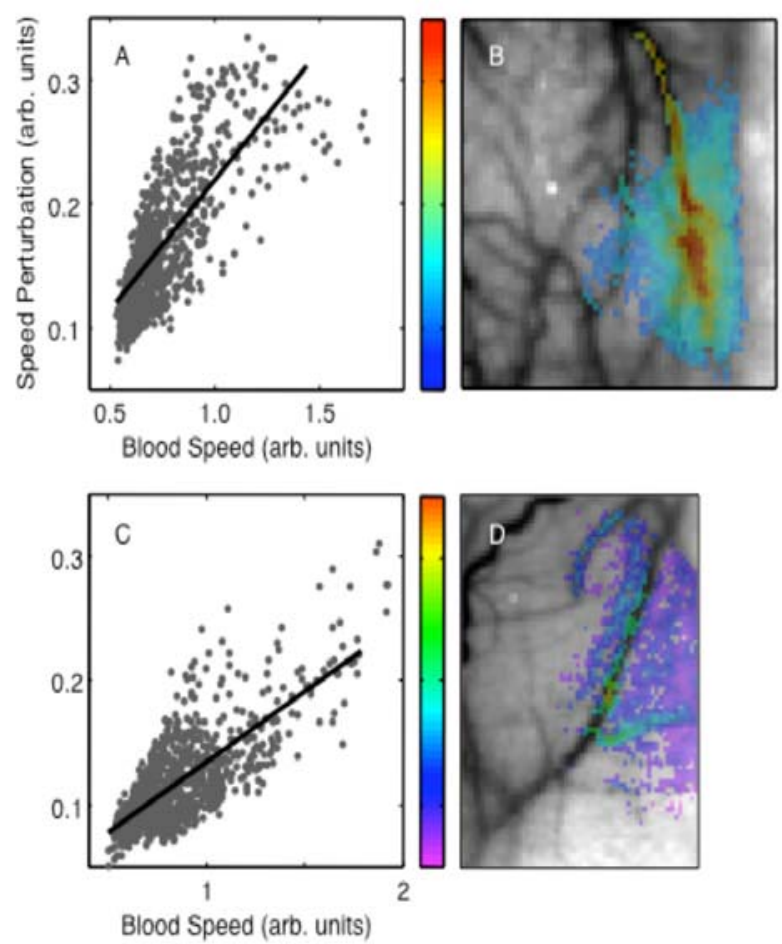

Fig. (4). Perturbation amplitude parameter: (A) spatial distribution (animal 1); (B) dependence on baseline blood speed (animal 1). Animal 2: (C), (D).
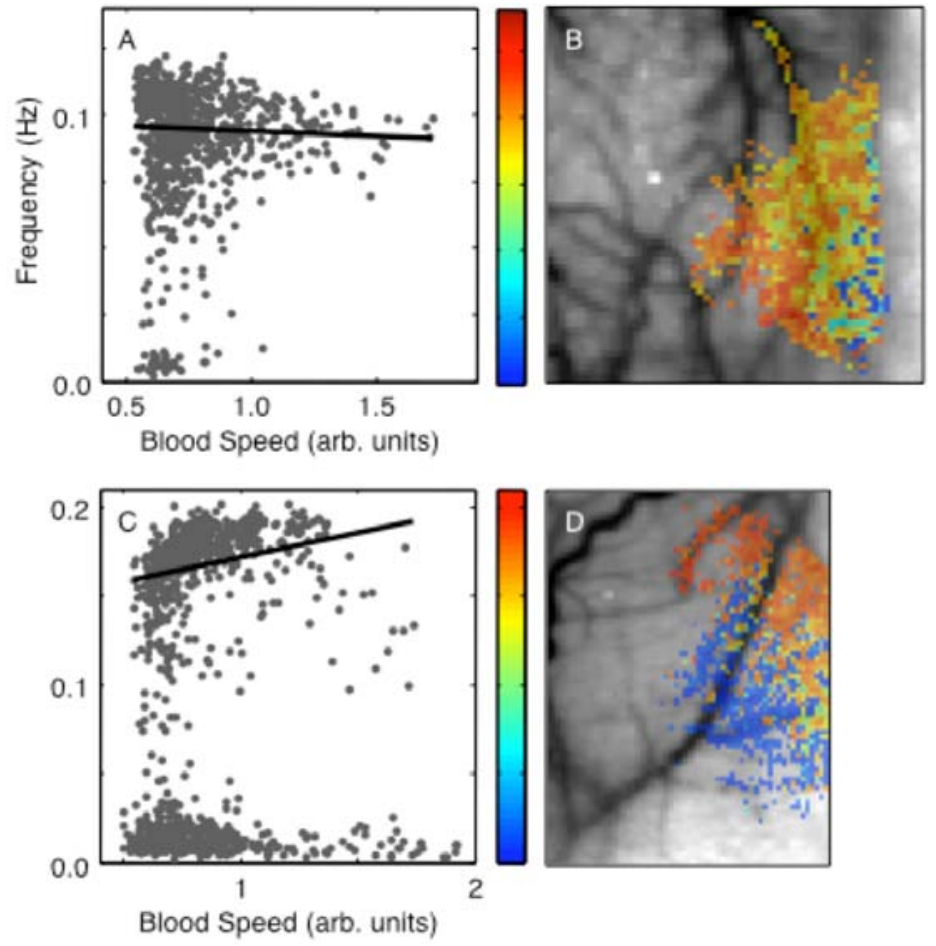

Fig. (5). Perturbation frequency parameter: (A) spatial distribution in animal 1; (B) dependence on baseline blood speed, animal 1. (C) and (D) show correspondingdata in animal 2.

amplitude and the baseline flow suggest that activation is, in fact, better revealed by a normalized flow metric. In animal 1, perturbation amplitude (Fig. 7A) suggests a region of activation toward the lower right that seems to follow the larger blood vessels. The normalized change in speed (Fig. 7B) illuminates a more spatially diffuse activation region.
This confirms and partially explains the utility of fractional signal change as a standard metric in fMRI and functional optical imaging data analysis.

We achieved good fits without modeling an initial period of latency or "initial dip" observed by other means. This suggests that the flow responses occurred promptly with 

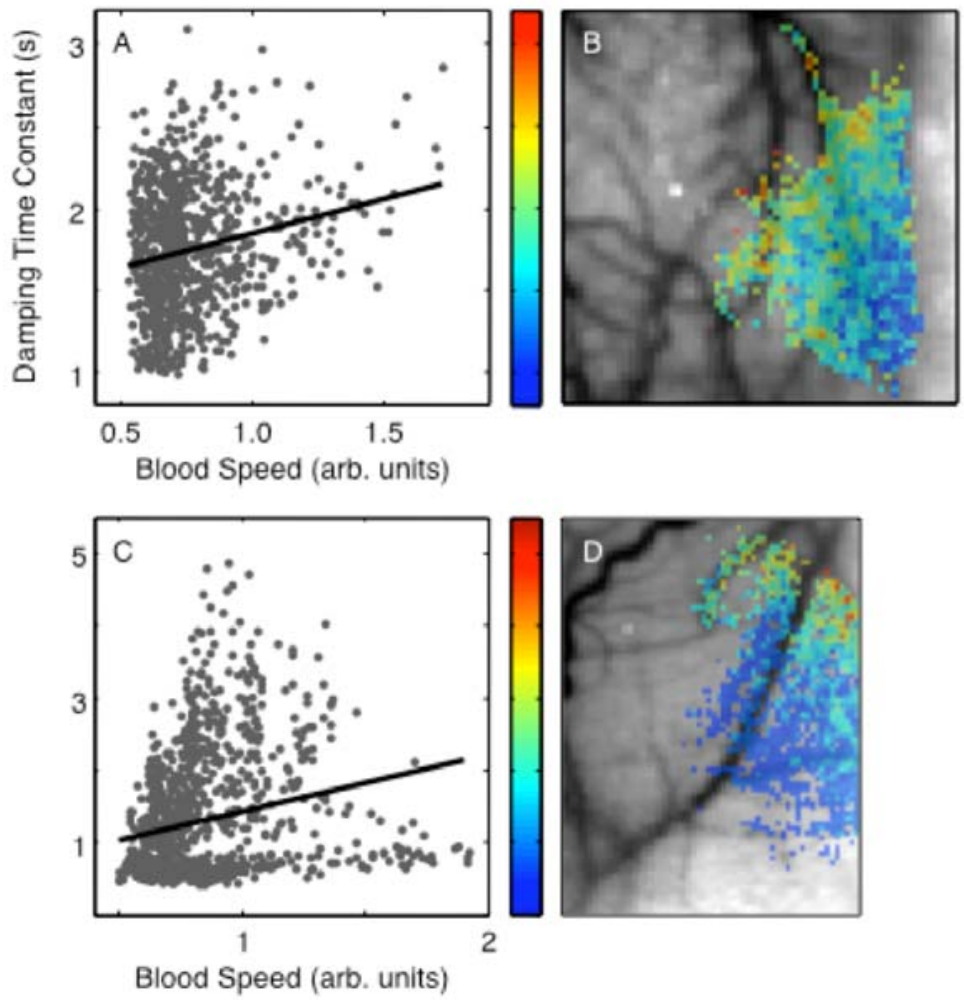

Fig. (6). Perturbation damping time parameter: (A) spatial distribution in animal 1; (B) dependence on baseline blood speed in animal 1. (C) and (D) show corresponding data for animal 2.
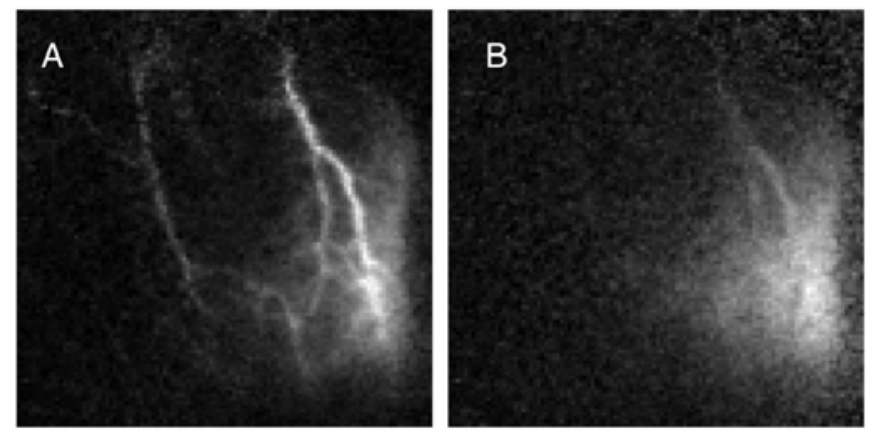

Fig. (7). Perturbation amplitude (A) compared to relative fluctuation level (B).

stimulation and are not associated with transit-time delays from remote control points. The mean frequency parameter in the underdamped regime, $\sim 0.08 \mathrm{~Hz}$, was quite stable, suggesting that the pial vascular network behaves as a second-degree, roughly linear filter that is mechanically tuned to a particular frequency. Moreover, the median damping time of $1.9-2.5 \mathrm{~s}$ suggests that this network has a slightly underdamped, bandpass character.

The frequency fits were relatively stable with baseline flow speed, decreasing only slightly over a large range of baseline flow speeds and perturbation amplitudes. The stability of this parameter suggests that both the inertance and compliance parameters of the vasculature are also relatively stable. The stability of the compliance is very reasonable, because the compliance "seen" within the microvasculature will be strongly dominated by the downstream compliance of venous tree, a global property. The inertance within the model corresponds to the mass of the blood regulated by the vascular control structures.
Stability of the inertance would suggest that the key regulatory structures are spaced in a consistent stereotypical fashion in the cerebral microvasculature, perhaps at the level of the pial penetrating arterioles.

In contrast, the damping time showed a significant tendency to rise with blood speed. This is consistent with viscous flow properties in the smaller vessels where flow speeds are low, transitioning to laminar flow in the larger vessels where flow speeds are higher.

The spatial distribution of the flow responses showed an interesting bipartite distribution, with less damped responses occurring in one region, typically close to the focus of the activation, and more strongly damped responses occurring in more weakly activated regions. These observations suggest that the underdamped responses are more prevalent in microvascular regions closer to the regulatory source of the perturbation, and these responses become more damped in distal, venular portions of the microvasculature. 
Our model did not as effectively fit the data observed in animal 2. This data appeared to exhibit a combination of a more strongly damped response, which was captured by our model, together with additional underdamped ringing at a higher frequency. This ringing is likely to be stimulus induced, because it is clearly evident and statistically significant after averaging over many trials. These data suggest the existence of more complex flow responses in response to repetitive transient stimulation. It is possible that such results could be modeled by more complex linear networks. For example, the measured flow response could be the superposition of flows mediated by two (or more) different mechanical regulation circuits, each with different time constants. Alternatively, the greater complexity could be introduced by neural feedback. On the other hand, these more complex responses could also be an artifact of the anesthesia, with little correspondence to the normal transient flow response.

Responses observed here were much more strongly damped than those observed in the tissue oxygen measurements obtained in cat visual cortex, $\sim 2.2 \mathrm{~s}$ here versus $\sim 9 \mathrm{~s}$ in cat [16]. The discrepancy could be explained in several ways. First, there could be delayed compliance effects that modify blood volume in a fashion distinct from flow changes. Delayed compliance has been a standard explanation used in many previous models [9-11, 13]. However, the absence of substantial volume changes in venular or capillary volume measurements [36] argues against this explanation because an increase in arterial blood volume alone cannot account for the large undershoots observed in both $\mathrm{HbR}$ and total $\mathrm{Hb}$ concentrations [3]. Another possibility is a species difference between rodents and cats. A third explanation concerns the level of anesthesia used in the experiments. In the cat experiments, anesthesia was maintained at a level light enough to permit significant neuronal activity as inferred by real-time EEG measurements. Such techniques were not used during the present experiments. The observed strongly damped responses, therefore, may have been caused by a different state of anesthesia. Our observation of more complex oscillatory responses in animal 3 suggests that neither the FEW model nor delayed compliance explanations may be sufficient to explain the HRF. Another possibility is that, for transient stimulation, visual cortex flow response is different, more underdamped, than that of somatosensory cortex. Further analysis and experiments will be necessary to establish the "normal" amount of ringing present in the flow response to brief neural activation.

The bandpass character of our flow model suggests an alternative explanation for some of the low-frequency oscillations or "vasomotion" observed with various techniques, e.g. [1, 32, 37-40]. Generally, these slow hemodynamic fluctuations have been attributed to mechanisms such as blood pressure feedback from baroreceptors or neurogenic mechanisms. These fluctuations, sometimes called Mayer waves, are observed in three frequency bands. The so-called "low-frequency" band of such fluctuations occurs at $\sim 0.1 \mathrm{~Hz}$, making it particularly relevant to the measurements and model presented here. The FEW has a bandpass response to transient changes in cerebrovascular control. Thus, unstimulated or spontaneous neural activity would be filtered by the hemodynamic network to appear as low-frequency oscillations in flow, oxygen transport, and other aspects of the hemodynamics. This hypothesis is consistent with the use of low-frequency fluctuations as a "resting-state" measurement of neural activity using fMRI [41-43]. The purpose of these oscillations is not yet understood. It has been suggested that low-frequency oscillations could increase oxygen transport efficacy $[44,45]$.

It could also be that several mechanisms are responsible for these fluctuations, with the FEW responsible only for fluctuations in hemodynamics associated with transient neural activation, while other neural feedback mechanisms, generally believed to driven by blood $\mathrm{CO}_{2}$ concentrations (e.g., [46]), are responsible for oxygen auto-regulatory functions. It is also possible that the two mechanisms are related, with the tuned bandpass character of the cerebral vasculature being actually a consequence of the putative neurogenic feedback mechanisms. Although in global scope, the mechanism of the low frequency fluctuations may be complex and non-linear [47], at the level of the present cerebrovascular oxygen measurements they may be satisfactorily described by the FEW linear model. Although the FEW model is a substantial simplification of the complex flow dynamics associated with the whole of the cerebrovasculature, within the context of linear network models this kind of analogy has been utilized extensively to solve many problems. Complex networks of linear mechanical or fluid elements can always be converted to a Norton or Thevenin equivalent with a multi-pole admittance or impedance [48], and this approach has been utilized extensively in cardiovascular applications $[8,49,50]$. In this view, the dynamics of the FEW are an abstract model of more complex feedback mechanisms rather than a literal model of simple mechanical processes. Further experiments will be necessary to elucidate the relationship between the FEW and low frequencyfluctuations.

\section{ACKNOWLEDGEMENTS}

We thank reviewer Junjie Liu for his many positive and helpful suggestions. Speckle measurement work was supported by the National Institutes of Health (EB008715, NS45879) and the American Heart Association (0735136N), and MERIT from the Department of Veterans Affairs.

\section{REFERENCES}

[1] Thompson JK, Peterson MR, Freeman RD. Single-neuron activity and tissue oxygenation in the cerebral cortex. Science 2003; 299(5609): 1070-2.

[2] Thompson JK, Peterson MR, Freeman RD. Separate spatial scales determine neural activity-dependent changes in tissue oxygen within central visual pathways. J Neurosci 2005; 25(39): 9046-58.

[3] Berwick J, Johnston D, Jones M, et al. Fine detail of neurovascular coupling revealed by spatiotemporal analysis of the hemodynamic response to single whisker stimulation in rat barrel cortex. J Neurophysiol 2008; 99(2): 787-98.

[4] Buxton RB, Uludag K, Dubowitz DJ, Liu TT. Modeling the hemodynamic response to brain activation. Neuroimage 2004; 23 (Suppl 1): S220-33.

[5] Buxton RB, Wong EC, Frank LR. Dynamics of blood flow and oxygenation changes during brain activation: the balloon model. Magn Reson Med 1998; 39(6): 855-64.

[6] Binzoni T, Quaresima V, Ferrari M, Hiltbrand E, Cerretelli P. Human calf microvascular compliance measured by near-infrared spectroscopy. J Appl Physiol 2000; 88(2): 369-72. 
[7] Romney JS, Lewanczuk RZ. Vascular compliance is reduced in the early stages of type 1 diabetes. Diabetes Care 2001; 24(12): 21026.

[8] Stergiopulos N, Westerhof BE, Westerhof N. Total arterial inertance as the fourth element of the windkessel model. Am J Physiol 1999; 276(1 Pt 2): H81-8.

[9] Mandeville JB, Marota JJ, Ayata C, et al. Evidence of a cerebrovascular postarteriole windkessel with delayed compliance. J Cereb Blood Flow Metab 1999; 19(6): 679-89.

[10] Friston KJ, Mechelli A, Turner R, Price CJ. Nonlinear responses in fMRI: the balloon model, volterra kernels, and other hemodynamics. Neuroimage 2000; 12(4): 466-77.

[11] Huppert TJ, Allen MS, Benav H, Jones PB, Boas DA. A multicompartment vascular model for inferring baseline and functional changes in cerebral oxygen metabolism and arterial dilation. J Cereb Blood Flow Metab 2007; 27(6): 1262-79.

[12] Zheng Y, Johnston D, Berwick J, Chen D, Billings S, Mayhew J. A three-compartment model of the hemodynamic response and oxygen delivery to brain. Neuroimage 2005; 28(4): 925-39.

[13] Zheng Y, Martindale J, Johnston D, Jones M, Berwick J, Mayhew J. A model of the hemodynamic response and oxygen delivery to brain. Neuroimage 2002; 16(3 Pt 1): 617-37.

[14] Vazquez AL, Fukuda M, Tasker ML, Masamoto K, Kim SG. Changes in cerebral arterial, tissue and venous oxygenation with evoked neural stimulation: implications for hemoglobin-based functional neuroimaging. J Cereb Blood Flow Metab 2010; 30(2): 428-39.

[15] Kim T, Hendrich KS, Masamoto K, Kim SG. Arterial versus total blood volume changes during neural activity-induced cerebral blood flow change: implication for BOLD fMRI. J Cereb Blood Flow Metab 2007; 27(6): 1235-47.

[16] Ress D, Thompson JK, Rokers B, Khan RK, Huk AC. A model for transient oxygen delivery in cerebral cortex. Front Neuroenergetics 2009; $1: 3$.

[17] Harrison RV, Harel N, Panesar J, Mount RJ. Blood capillary distribution correlates with hemodynamic-based functional imaging in cerebral cortex. Cereb Cortex 2002; 12(3): 225-33.

[18] Peppiatt CM, Howarth C, Mobbs P, Attwell D. Bidirectional control of CNS capillary diameter by pericytes. Nature 2006; 443(7112): 700-4.

[19] Rodriguez-Baeza A, Reina-De La Torre F, Ortega-Sanchez M, Sahuquillo-Barris J. Perivascular structures in corrosion casts of the human central nervous system: a confocal laser and scanning electron microscope study. Anat Rec 1998; 252(2): 176-84.

[20] Iadecola C, Nedergaard M. Glial regulation of the cerebral microvasculature. Nat Neurosci 2007; 10(11): 1369-76.

[21] Martin C, Martindale J, Berwick J, Mayhew J. Investigating neuralhemodynamic coupling and the hemodynamic response function in the awake rat. Neuroimage 2006; 32(1): 33-48.

[22] Martindale J, Mayhew J, Berwick J, et al. The hemodynamic impulse response to a single neural event. J Cereb Blood Flow Metab 2003; 23(5): 546-55.

[23] Masamoto K, Kim T, Fukuda M, Wang P, Kim SG. Relationship between neural, vascular, and BOLD signals in isofluraneanesthetized rat somatosensory cortex. Cereb Cortex 2007; 17(4): 942-50.

[24] Durduran T, Burnett MG, Yu G, et al. Spatiotemporal quantification of cerebral blood flow during functional activation in rat somatosensory cortex using laser-speckle flowmetry. J Cereb Blood Flow Metab 2004; 24(5): 518-25.

[25] Dunn AK, Bolay H, Moskowitz MA, Boas DA. Dynamic imaging of cerebral blood flow using laser speckle. J Cereb Blood Flow Metab 2001; 21(3): 195-201.

[26] Dunn AK, Devor A, Bolay H, et al. Simultaneous imaging of total cerebral hemoglobin concentration, oxygenation, and blood flow during functional activation. Opt Lett 2003; 28(1): 28-30.

[27] Dunn AK, Devor A, Dale AM, Boas DA. Spatial extent of oxygen metabolism and hemodynamic changes during functional activation of the rat somatosensory cortex. Neuroimage 2005; 27(2): 279-90.
[28] Tom WJ, Ponticorvo A, Dunn AK. Efficient processing of laser speckle contrast images. IEEE Trans Med Imaging 2008; 27(12): 1728-38.

[29] Fercher AF, Briers JD. Flow visualization by means of singleexposure speckle photography. Optics Communications 1981; 37(5): 326-30.

[30] Bonner R, Nossal R. Model for laser Doppler measurements of blood flow in tissue. Appl Opt 1981; 20(12): 2097-107.

[31] Jakobsson A, Nilsson GE. Prediction of sampling depth and photon pathlength in laser Doppler flowmetry. Med Biol Eng Comput 1993; 31(3): 301-7.

[32] Kleinfeld D, Mitra PP, Helmchen F, Denk W. Fluctuations and stimulus-induced changes in blood flow observed in individual capillaries in layers 2 through 4 of rat neocortex. Proc Natl Acad Sci USA 1998; 95(26): 15741-6.

[33] Coleman TF, Li Y. An Interior Trust Region Approach for Nonlinear Minimization Subject to Bounds. SIAM Journal on Optimization 1996; 6(2): 418-45.

[34] Fox PT, Raichle ME. Stimulus rate dependence of regional cerebral blood flow in human striate cortex, demonstrated by positron emission tomography. J Neurophysiol 1984; 51(5): 1109-20.

[35] Feng CM, Narayana S, Lancaster JL, et al. CBF changes during brain activation: fMRI vs. PET. Neuroimage 2004; 22(1): 443-6.

[36] Lee SP, Duong TQ, Yang G, Iadecola C, Kim SG. Relative changes of cerebral arterial and venous blood volumes during increased cerebral blood flow: implications for BOLD fMRI. Magn Reson Med 2001; 45(5): 791-800.

[37] Katura T, Tanaka N, Obata A, Sato H, Maki A. Quantitative evaluation of interrelations between spontaneous low-frequency oscillations in cerebral hemodynamics and systemic cardiovascular dynamics. Neuroimage 2006; 31(4): 1592-600.

[38] Koepchen HP. History of studies and concepts of blood pressure waves. In: K. Miyakawa HPK, and C. Polosa, editor. Mechanisms of Blood Pressure Waves. New York: Springer-Verlag; 1984. p. 327.

[39] Mayhew JE, Askew S, Zheng Y, et al. Cerebral vasomotion: a 0.1$\mathrm{Hz}$ oscillation in reflected light imaging of neural activity. Neuroimage 1996; 4(3 Pt 1): 183-93.

[40] Spitzer MW, Calford MB, Clarey JC, Pettigrew JD, Roe AW. Spontaneous and stimulus-evoked intrinsic optical signals in primary auditory cortex of the cat. J Neurophysiol 2001; 85(3): 1283-98.

[41] Biswal BB, Van Kylen J, Hyde JS. Simultaneous assessment of flow and BOLD signals in resting-state functional connectivity maps. NMR Biomed 1997; 10(4-5): 165-70.

[42] Peltier SJ, Noll DC. T(2)(*) dependence of low frequency functional connectivity. Neuroimage 2002; 16(4): 985-92.

[43] Fransson P. Spontaneous low-frequency BOLD signal fluctuations: an fMRI investigation of the resting-state default mode of brain function hypothesis. Hum Brain Mapp 2005; 26(1): 15-29.

[44] Goldman D, Popel AS. A computational study of the effect of vasomotion on oxygen transport from capillary networks. J Theor Biol 2001; 209(2): 189-99.

[45] Kislukhin VV. Regulation of oxygen consumption by vasomotion. Math Biosci 2004; 191(1): 101-8.

[46] Kety SS, Schmidt CF. The effects of altered arterial tensions of carbon dioxide and oxygen on cerebral blood flow and cerebral oxygen consumption of normal young men. J Clin Invest 1948; 27(4): 484-92.

[47] Seydnejad SR, Kitney RI. Modeling of Mayer waves generation mechanisms. IEEE Eng Med Biol Mag 2001; 20(2): 92-100.

[48] Olson HF. Dynamical Analogies, 1st ed. New York: D. Van Nostrand Company, Inc.; 1943.

[49] Burattini R, Di Salvia PO. Development of systemic arterial mechanical properties from infancy to adulthood interpreted by four-element windkessel models. J Appl Physiol 2007; 103(1): 6679 .

[50] Thiry PS, Roberge FA. [Analogs and models of systemic arterial circulation]. Rev Can Biol 1976; 35(4): 217-38. 